\title{
Hybrid Systems with Finite Bisimulations ${ }^{\star}$
}

\author{
Gerardo Lafferriere $^{1}$, George J. Pappas ${ }^{2}$, and Shankar Sastry ${ }^{2}$ \\ 1 Department of Mathematical Sciences \\ Portland State University, Portland, OR 97207, USA \\ gerardo@mth.pdx.edu \\ 2 Department of Electrical Engineering and Computer Sciences \\ University of California at Berkeley, Berkeley, CA 94720, USA \\ \{gpappas, sastry\}@eecs. berkeley.edu
}

\begin{abstract}
The theory of formal verification is one of the main approaches to hybrid system analysis. Decidability questions for verification algorithms are obtained by constructing finite, reachability preserving quotient systems called bisimulations. In this paper, we use recent results from stratification theory, subanalytic sets, and model theory in order to extend the state-of-the-art results on the existence of bisimulations for certain classes of planar hybrid systems.
\end{abstract}

\section{Introduction}

Hybrid systems consist of finite state machines interacting with differential equations. Various modeling formalisms, analysis, design and control methodologies, as well as applications, can be found in 3445/10]17. Formal verification is the main computational approach for analyzing properties of hybrid systems. One of the most important verification problems for hybrid systems is the reachability problem which asks whether trajectories of the hybrid system can reach certain undesirable regions of the state space. Since hybrid systems have infinite state spaces, the decidability of verification algorithms is very important.

A uniform framework for tackling the decidability issue is provided by the notion of bisimulation. Bisimulations are quotients of the original hybrid system that are reachability preserving. Showing that an infinite state hybrid system has a finite state bisimulation is the first step in proving decidability. This approach has been successfully applied to timed automata [2, multirate automata [1], and initialized rectangular automata 19:12. It should be mentioned that the notion of bisimulation is closely related to the various consistency notions for discrete and continuous systems [7818].

Since the discrete dynamics are already finite, it is clear that decidability results for hybrid systems depend crucially on the success of obtaining finite bisimulations for continuous dynamics. The cases considered so far in the literature dealt with simple dynamics: $\dot{x}=1$ for timed automata [2], $\dot{x}=a$ for

\footnotetext{
* Research supported by the Army Research Office under grants DAAH 04-95-1-0588 and DAAH 04-96-1-0341.
} 
multirate automata [1], $\dot{x} \in[a, b]$ for rectangular automata [19], and $A \dot{x} \leq b$ for linear hybrid automata [11. In this paper, we extend the bisimulation methodology to hybrid systems with more general dynamics. We first present the standard bisimulation algorithm which, upon termination, provides the desired finite bismilarity quotient. In [15, we used purely geometric methods to show that the bisimulation algorithm terminates for a class of hybrid systems with planar linear dynamics. In this paper, we combine mathematical techniques from differential geometry and recent results in logic model theory in ordet to prove existence of finite bisimulations for various new classes of hybrid systems with planar continuous dynamics. This convergence of mathematical logic and differential geometry also provides a natural framework for extending the decidability frontier for more general classes of hybrid systems. Such extensions will require pushing the boundary of decidable theories in mathematical logic.

Abstracting a discrete graph from a hybrid system requires the analysis of trajectories of vector fields and their intersection properties relative to a given collection of sets. Considering hybrid systems with arbitrary dynamics and arbitrary state partitions would soon lead to pathological situations. Subanalytic sets 6 [1321] provide a rich class of sets which have many desirable local intersection properties with trajectories of analytic vector fields. Subanalytic sets can also be partitioned into smooth embedded submanifolds in a form suitable for constructing a bisimulation. Such partitions are called stratifications. Moreover, we show that relaxing the class of vector fields or sets in some naive ways leads to pathological situations. On the other hand, the concept of o-minimal theories in logic [24|25 26] identifies classes of sets with good intersection properties suitable for the global study of trajectories of vector fields. The combination of techniques from both fields highlights the kind of properties of sets that play a central role in obtaining discrete abstractions.

The outline of the paper is as follows: In Section 2 we review the notion of bisimulations of transitions systems. In Section 3 we define the class of hybrid systems under study and describe the main bisimulation algorithm. Section 4 presents some basic facts about stratification theory and subanalytic sets and relates them to the construction of bisimulations. In Section 5 we present recent results in model theory which are used in Section 6 in order to obtain classes of systems for which the bisimulation algorithm terminates. Section 7 contains conclusions and issues for further research.

\section{Bisimulations of Transition Systems}

A transition system $T=\left(Q, \Sigma, \rightarrow, Q_{O}, Q_{F}\right)$ consists of a (not necessarily finite) set $Q$ of states, an alphabet $\Sigma$ of events, a transition relation $\rightarrow \subseteq Q \times \Sigma \times Q$, a set $Q_{O} \subseteq Q$ of initial states, and a set $Q_{F} \subseteq Q$ of final states. A transition $\left(q_{1}, \sigma, q_{2}\right) \in \rightarrow$ is denoted as $q_{1} \stackrel{\sigma}{\rightarrow} q_{2}$. The transition system is finite if the cardinality of $Q$ is finite and it is infinite otherwise. A region is a subset $P \subseteq Q$. Given $\sigma \in \Sigma$ we define the predecessor $\operatorname{Pre}_{\sigma}(P)$ of a region $P$ as

$$
\operatorname{Pre}_{\sigma}(P)=\{q \in Q \mid \exists p \in P \text { and } q \stackrel{\sigma}{\rightarrow} p\}
$$


Given an equivalence relation $\sim \subseteq Q \times Q$, we define a quotient transition system $t / \sim$ as follows: Let $Q / \sim$ denote the quotient space. For a region $P$ we denote by $P / \sim$ the collection of all equivalence classes which intersect $P$. The transition relation $\rightarrow \sim$ on the quotient space is defined as follows: for $Q_{1}, Q_{2} \in Q / \sim$, $Q_{1} \stackrel{\sigma}{\rightarrow} \sim Q_{2}$ iff there exist $q_{1} \in Q_{1}$ and $q_{2} \in Q_{2}$ such that $q_{1} \stackrel{\sigma}{\rightarrow} q_{2}$. The quotient transition system is then $T / \sim=\left(Q / \sim, \Sigma, \rightarrow \sim, Q_{0} / \sim, Q_{F} / \sim\right)$.

Given a partition $\sim$ on $Q$, we call a set a $\sim$-block if it is a union of equivalence classes. The partition $\sim$ is a bisimulation of $T$ iff $Q_{O}, Q_{F}$ are $\sim$-blocks and for all $\sigma \in \Sigma$ and all $\sim$-blocks $P$, the region $\operatorname{Pre}_{\sigma}(P)$ is a $\sim$-block. In this case the systems $T$ and $T / \sim$ are called bisimilar. A bisimulation is called finite if it has a finite number of equivalence classes. Bisimulations are very important because checking reachability on the bisimilar transition system is equivalent to checking reachability of the original transition system [11. Therefore, if $T$ is infinite and $T / \sim$ is a finite bisimulation, then verification algorithms for infinite systems are guaranteed to terminate. If $\sim$ is a bisimulation, it can be easily shown that if $p \sim q$ then

B1 $p \in Q_{\sigma}$ iff $q \in Q_{F}$, and $p \in Q_{O}$ iff $q \in Q_{O}$

B2 if $p \stackrel{\sigma}{\rightarrow} p^{\prime}$ then there exists $q^{\prime}$ such that $q \stackrel{\sigma}{\rightarrow} q^{\prime}$ and $p^{\prime} \sim q^{\prime}$

Based on the above characterization, given a transition system $T$, the following algorithm computes increasingly finer partitions of the state space $Q$.

Algorithm 1 (Bisimulation Algorithm for Transition Systems)

Set $Q / \sim=\left\{Q_{O} \cap Q_{F}, Q_{O} \backslash Q_{F}, Q_{F} \backslash Q_{O}, Q \backslash\left(Q_{O} \cup Q_{F}\right)\right\}$

while $\exists P, P^{\prime} \in Q / \sim$ and $\sigma \in \Sigma$ such that $\emptyset \neq P \cap \operatorname{Pre}_{\sigma}\left(P^{\prime}\right) \neq P$

set $P_{1}=P \cap \operatorname{Pre}_{\sigma}\left(P^{\prime}\right), P_{2}=P \backslash \operatorname{Pre}_{\sigma}\left(P^{\prime}\right)$

refine $Q / \sim=(Q / \sim \backslash\{P\}) \cup\left\{P_{1}, P_{2}\right\}$

\section{end while}

If the algorithm terminates, then the resulting quotient transition system is a finite bisimulation. The goal of the next sections is to show that the above algorithm terminates for transition systems generated by a class of planar hybrid systems.

\section{Bisimulations of Hybrid Systems}

In this paper, we focus on transition systems generated by the following class of hybrid systems.

Definition 1. A hybrid system $H=\left(X, X_{0}, X_{F}, F, E, I, G, R\right)$ consists of

- $X=X_{D} \times X_{C}$ is the state space with $X_{D}=\left\{q_{1}, \ldots, q_{n}\right\}$ and $X_{C}$ an analytic manifold.

$-X_{0} \subseteq X$ is the set of initial states

$-X_{F} \subseteq X$ is the set of final states

$-F: X \longrightarrow T X_{C}$ assigns to each discrete state $q \in X_{D}$ an analytic vector field $F(q, \cdot)$ 
$-E \subseteq X_{D} \times X_{D}$ is the set of discrete transitions

$-I: X_{D} \longrightarrow 2^{X_{C}}$ assigns to each discrete state a set $I(q) \subseteq X_{C}$ called the invariant.

- $G: E \longrightarrow X_{D} \times 2^{X_{C}}$ assigns to $e=\left(q_{1}, q_{2}\right) \in E$ a guard of the form $\left\{q_{1}\right\} \times U$, $U \subseteq I\left(q_{1}\right)$.

$-R: E \longrightarrow X_{D} \times 2^{X_{C}}$ assigns to $e=\left(q_{1}, q_{2}\right) \in E$ a reset of the form $\left\{q_{2}\right\} \times V$, $V \subseteq I\left(q_{2}\right)$.

Trajectories of $H$ start at any $(q, x) \in X_{0}$ and consist of continuous evolutions or discrete jumps. Continuous trajectories evolve according to the continuous flow $F(q, \cdot)$ as long as the state remains in the invariant set $I(q)$. If the flow exits $I(q)$, then a discrete transition is forced. If, during the continuous evolution, the state $(q, x)$ enters a region $G(e)$ for some $e \in E$, then discrete transition $e$ is enabled, and the state may instantaneously jump from $(q, x)$ to any $\left(q^{\prime}, x^{\prime}\right) \in R(e)$. Then the system evolves according to $F\left(q^{\prime}, \cdot\right)$. Notice that the discrete transitions allowed in our model are similar to the type allowed in initialized rectangular automata [19]. Finally, we assume that our hybrid system model is non-blocking, that is from every state either a continuous evolution or a discrete transition is possible.

Every hybrid system $H=\left(X, X_{0}, X_{F}, F, E, I, G, R\right)$ generates a transition system $T=\left(Q, \Sigma, \rightarrow, Q_{O}, Q_{F}\right)$ by setting $Q=X, Q_{0}=X_{0}, Q_{F}=X_{F}, \Sigma=$ $E \cup\{\tau\}$, and $\rightarrow=\left(\cup_{e \in E} \stackrel{e}{\rightarrow}\right) \cup \stackrel{\tau}{\rightarrow}$ where

Discrete Transitions $(q, x) \stackrel{e}{\rightarrow}\left(q^{\prime}, x^{\prime}\right)$ for $e \in E$ iff $(q, x) \in G(e)$ and $\left(q^{\prime}, x^{\prime}\right) \in$ $R(e)$

Continuous Transitions $\left(q_{1}, x_{1}\right) \stackrel{\tau}{\rightarrow}\left(q_{2}, x_{2}\right)$ iff $q_{1}=q_{2}$ and there exists $\delta \geq 0$ and a curve $x:[0, \delta] \longrightarrow M$ with $x(0)=x_{1}, x(\delta)=x_{2}$ and for all $t \in[0, \delta]$ it satisfies $x^{\prime}=F\left(q_{1}, x(t)\right)$ and $x(t) \in I\left(q_{1}\right)$.

The continuous $\tau$ transitions are time-abstract transitions, in the sense that the time it takes to reach one state from another is ignored. Having defined the continuous and discrete transitions $\stackrel{\tau}{\rightarrow}$ and $\stackrel{e}{\rightarrow}$ allows us to formally define $\operatorname{Pre}_{\tau}(P)$ and $\operatorname{Pre}_{e}(P)$ for $e \in E$ and any region $P \subseteq X$ using (1). Furthermore, the discrete transitions allowed in our hybrid system model result in

$$
\operatorname{Pre}_{e}(P)= \begin{cases}\emptyset & \text { if } P \cap R(e)=\emptyset \\ G(e) & \text { if } P \cap R(e) \neq \emptyset\end{cases}
$$

for all discrete transitions $e \in E$ and regions $P$. Therefore, if the sets $R(e)$ and $G(e)$ are blocks of any partition of the state space, then no partition refinement is necessary in the bisimulation algorithm due to any discrete transitions $e \in$ $E$. This fact, decouples the continuous and discrete components of the hybrid system as long as the initial partition in the bisimulation algorithm contain the invariants, guards and reset sets, in addition to the initial and final sets. This allows us to carry out the bisimulation algorithm independently for each discrete state. 
More precisely, define for any region $P \subseteq X$ and $q \in X_{D}$ the set $P_{q}=\{x \in$ $\left.X_{C}:(q, x) \in P\right\}$. For each discrete state $q \in X_{D}$ consider the finite collection of sets

$$
\mathcal{A}_{q}=\left\{I(q),\left(X_{0}\right)_{q},\left(X_{F}\right)_{q}\right\} \cup\left\{G(e)_{q}, R(e)_{q}: e \in E\right\}
$$

which describes all the relevant sets associated with discrete state $q$. Let $\mathcal{S}_{q}$ be the coarsest partition of $X_{C}$ compatible with the collection $\mathcal{A}_{q}$ (by compatible we mean that each set in $\mathcal{A}_{q}$ is a union of sets in $\mathcal{S}_{q}$ ). The (finite) partition $\mathcal{S}_{q}$ can be easily computed by successively finding the intersections between each of the sets in $\mathcal{A}_{q}$ and their complements. These collections $\mathcal{S}_{q}$ will be the starting partitions of the following bisimulation algorithm for hybrid systems.

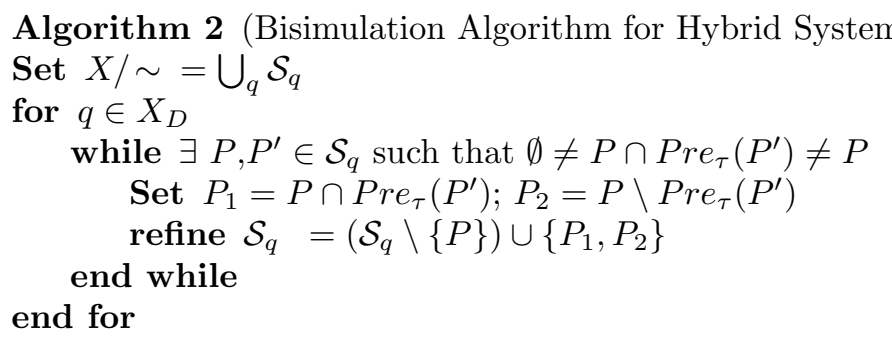

A few comments are in order here. The key problem is to investigate how the flow of $F(q, \cdot)$ interacts with the sets $\mathcal{S}_{q}$ for a single discrete state $q$. This requires that the trajectories of the vector field $F(q, \cdot)$ have "nice" intersection properties with such sets. Since the goal is to obtain finite partitions, it will become important that we restrict the study to classes of sets with good "finiteness" properties, for example, sets with finitely many connected components. In the subsequent sections we identify classes of sets and vector fields which exhibit such properties and for which Algorithm 2 terminates.

One can also view the partitions in the algorithm as a way of discretizing the system trajectories. This suggests studying the continuous transitions by looking only at the points at which the trajectories move from one set in $\mathcal{S}_{q}$ to an "adjacent" one. This is in general not possible because sets could have rather pathological boundaries (see also Example 4). We will see in the next section that subanalytic sets are free from such pathologies and that in fact one can formalize the idea of trajectory discretization associated to the partition in that case.

We conclude this section with an example that shows that, even in apparently simple situations, Algorithm 2 does not terminate.

Example 1. Let $F$ be the linear vector field $\left(\begin{array}{cc}-1 & 1 \\ -1 & -1\end{array}\right) \mathbf{x}$ on $\mathbb{R}^{2}$. Assume the partition of $\mathbb{R}^{2}$ consists of the following three sets (see Figure 1): $P_{1}=\{(x, 0)$ : $0 \leq x \leq 4\}, P_{2}=\{(x, 0):-4 \leq x<0\}, P_{3}=\mathbb{R}^{2} \backslash\left(P_{1} \cup P_{2}\right)$. The integral curves of $F$ are spirals moving away from the origin. The first iteration of the algorithm partitions $P_{2}$ into $P_{4}=P_{2} \cap \operatorname{Pre}_{\tau}\left(P_{1}\right)=\left\{(x, 0): x_{1} \leq x<0\right\}$ and 


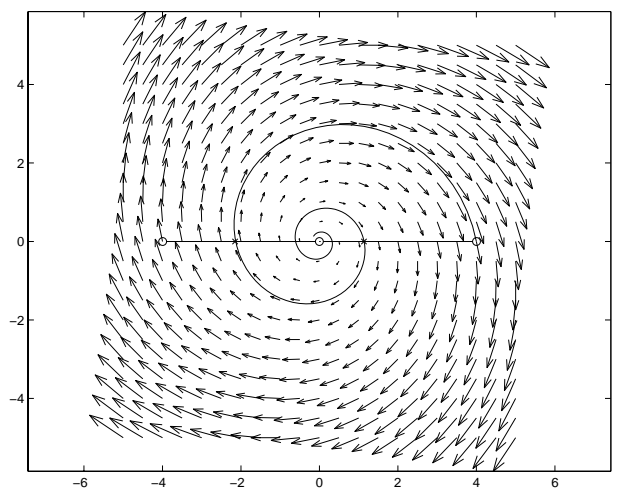

Fig. 1. Algorithm 2 does not terminate

$P_{2} \backslash \operatorname{Pre}_{\tau}\left(P_{1}\right)$. Here $x_{1}<0$ is the $x$-coordinate of the first intersection point of the spiral through $(4,0)$ with $P_{2}$. The second iteration subdivides $P_{1}$ into $P_{5}=P_{1} \cap \operatorname{Pre}_{\tau}\left(P_{4}\right)=\left\{(x, 0): 0 \leq x \leq x_{2}\right\}$ and $P_{1} \backslash \operatorname{Pre}_{\tau}\left(P_{4}\right)$ where $x_{2}>0$ is the $x$-coordinate of the next point of intersection of the spiral with $P_{1}$. This process continues indefinitely since the spiral intersects $P_{1}$ in infinitely many points, and therefore the algorithm does not terminate.

\section{Subanalytic Sets and Stratifications}

In this section we describe some fundamental properties of subanalytic sets (see 61321 for more details). A differentiable manifold is real analytic $\left(C^{\omega}\right)$ if the transition maps between local coordinate charts are analytic functions on their domains (which are open subsets of $\mathbb{R}^{n}$ ). An embedded submanifold $S$ of a manifold $M$ is a topological subspace of $M$ together with a differentiable structure such that the inclusion from $S$ into $M$ is a smooth immersion (i.e. has full rank at every point). A vector field $F$ on the real analytic manifold $M$ is analytic if its coordinates in any local chart are analytic. If $F$ is an analytic vector field then any integral curve of $F$ is analytic.

Let $M$ and $N$ be real analytic manifolds and let $C^{\omega}(M, N)$ denote the set of analytic functions from $M$ into $N$. If $f \in C^{\omega}(M, N)$ we say $f$ is of class $C^{\omega}$. Given an analytic manifold $U$, we denote by $\Sigma\left(C^{\omega}(U, \mathbb{R})\right)$ the Boolean algebra generated by the sets of the form $\{x: f(x)=0\}$ or $\{x: f(x)>0\}$, where $f \in C^{\omega}(U, \mathbb{R})$.

Definition 2. Let $M$ be a real analytic manifold. A subset $A$ of $M$ is semianalytic in $M$ if for every $p \in M$, there is an open neighborhood $U$ of $p$ in $M$ such that $U \cap A \in \Sigma\left(C^{\omega}(U, \mathbb{R})\right)$. If $A \subseteq M$ is semianalytic in $M$ we write $A \in \operatorname{SMAN}(M)$. 
Definition 3. Let $M$ be a real analytic manifold. Define $\operatorname{SBAN}_{r c}(M)$ and $\operatorname{SBAN}(M)$ by

1. $A \in \operatorname{SBAN}_{r c}(M)$ if and only if there is $\left(N, f, A^{*}\right)$ such that $N$ is a real analytic manifold, $f \in C^{\omega}(N, M), A^{*} \in \operatorname{SMAN}(N), A^{*}$ is relatively compact and $A=f\left(A^{*}\right)$;

2. $A \in \operatorname{SBAN}(M)$ if and only if $A$ is the union of a locally finite collection of members of $\operatorname{SBAN}_{r c}(M)$. (A collection of sets $\mathcal{C}$ is locally finite if any compact set intersect only finitely many sets in $\mathcal{C}$.)

We say that $A$ is subanalytic in $M$ if $A \in \operatorname{SBAN}(M)$. It is easy to see that $A \in \operatorname{SBAN}_{r c}(M)$ if and only if $A$ is subanalytic in $M$ and relatively compact. The following properties of subanalytic sets are easily derived from the definitions.

1. $\operatorname{SBAN}(M)$ is closed under locally finite unions and intersections.

2. If $A \in \operatorname{SBAN}(M)$ and $f: M \longrightarrow N$ is of class $C^{\omega}$ and proper on $\bar{A}$, the closure of $A$, then $f(A) \in \operatorname{SBAN}(N)$. (A function $f$ is proper if $f^{-1}(K)$ is compact whenever $K$ is.)

3. If $A \in \operatorname{SBAN}(N)$ and $f: M \longrightarrow N$ is of class $C^{\omega}$, then $f^{-1}(A) \in \operatorname{SBAN}(M)$.

The following two properties require more subtle proofs, but they give the first indication that this will be a suitable class of sets for our studies.

4. If $A \in \operatorname{SBAN}(M)$ then $M \backslash A \in \operatorname{SBAN}(M)$.

5. A subanalytic set has a locally finite number of connected components, each of which is subanalytic.

Example 2. Points are subanalytic, and so is any locally finite union of points, for example $\mathbb{Z}^{n}$ as subset of $\mathbb{R}^{n}$. The empty set and $M$ are both in $\operatorname{SBAN}(M)$. Let $a, b \in \mathbb{R}, a<b$, then $[a, b],[a, b),(a, b]$ and $(a, b)$ are subanalytic in $\mathbb{R}$. The open ball $B(p, r)$ centered at $p$ of radius $r$ in $\mathbb{R}^{n}$ is in $\operatorname{SBAN}\left(\mathbb{R}^{n}\right)$.

Definition 4. Let $M$ be a real analytic manifold. An analytic $\left(C^{\omega}\right)$ stratification of $M$ is a partition $\mathcal{S}$ of $M$ with the following properties:

1. each $S \in \mathcal{S}$ is a connected, real analytic, embedded submanifold of $M$,

2. $\mathcal{S}$ is locally finite,

3. given two sets $S, P \in \mathcal{S}, P \neq S$, such that $S \cap \bar{P} \neq \emptyset$ then $S \subset \bar{P}$ and $\operatorname{dim} S<\operatorname{dim} P$.

The sets in a stratification are called strata.

The central result on stratifications for our analysis is the following. For a proof see [20].

Theorem 1. Let $\mathcal{A}$ be a locally finite family of nonempty subanalytic subsets of a real analytic manifold $M$. For each $A \in \mathcal{A}$, let $F(A)$ be a finite set of real analytic vector fields on $M$. Then there exists a subanalytic stratification $\mathcal{S}$ of $M$, compatible with $\mathcal{A}$, and having the property that, whenever $S \in \mathcal{S}, S \subseteq A$, $A \in \mathcal{A}, X \in F(A)$, then either (i) $F$ is everywhere tangent to $S$ or (ii) $F$ is nowhere tangent to $S$. ( $\mathcal{S}$ is compatible with $\mathcal{A}$ is every set in $\mathcal{A}$ is a union of sets in $\mathcal{S}$.) 
Theorem 1 is illustrated by the following example.

Example 3. Let $F$ be the following analytic vector field on $\mathbb{R}^{2}$

$$
\begin{aligned}
& \dot{x}=x^{2}+y^{2} \\
& \dot{y}=0
\end{aligned}
$$

which has an isolated equilibrium at the origin and points in the positive $x$ direction otherwise. Consider the following two subanalytic sets

$$
\begin{array}{lll}
S_{1}=\left\{(x, y) \in \mathbb{R}^{2} \mid y \geq 0\right. & \text { and } & \left.(x-1)^{2}+y^{2}=1\right\} \\
S_{2}=\left\{(x, y) \in \mathbb{R}^{2} \mid y=0\right. & \text { and } & 0 \leq x \leq 2\}
\end{array}
$$

shown in Figure 2. A subanalytic stratification of $\mathbb{R}^{2}$ which is compatible with the sets $S_{1}, S_{2}$ and the vector field $F$ is also shown in Figure 2. It consists of

- 0-dimensional strata

- $P_{1}=(0,0), P_{2}=(2,0)$, and $P_{3}=(1,1)$

- 1-dimensional strata

- $C_{1}=\left\{(x, y) \in \mathbb{R}^{2} \mid y=0 \quad\right.$ and $\left.\quad 0<x<2\right\}$

- $C_{2}=\left\{(x, y) \in \mathbb{R}^{2} \mid y>0\right.$ and $1<x<2 \quad$ and $\left.\quad(x-1)^{2}+y^{2}=1\right\}$

- $C_{3}=\left\{(x, y) \in \mathbb{R}^{2} \mid y>0 \quad\right.$ and $\quad 0<x<1 \quad$ and $\left.\quad(x-1)^{2}+y^{2}=1\right\}$

- 2-dimensional strata

- $D_{1}=\left\{(x, y) \in \mathbb{R}^{2} \mid y>0\right.$ and $\left.(x-1)^{2}+y^{2}<1\right\}$

- $D_{2}=\mathbb{R}^{2} \backslash\left\{P_{1}, P_{2}, P_{3}, C_{1}, C_{2}, C_{3}, D_{1}\right\}$

Notice that the vector field is tangent to $P_{1}$ since it is an equilibrium as well as to $C_{1}, D_{1}$ and $D_{2}$. The vector field is transverse to all the other strata. Moreover, $S_{1}=P_{1} \cup P_{2} \cup P_{3} \cup C_{2} \cup C_{3}$ and $S_{2}=P_{1} \cup P_{2} \cup C_{1}$.
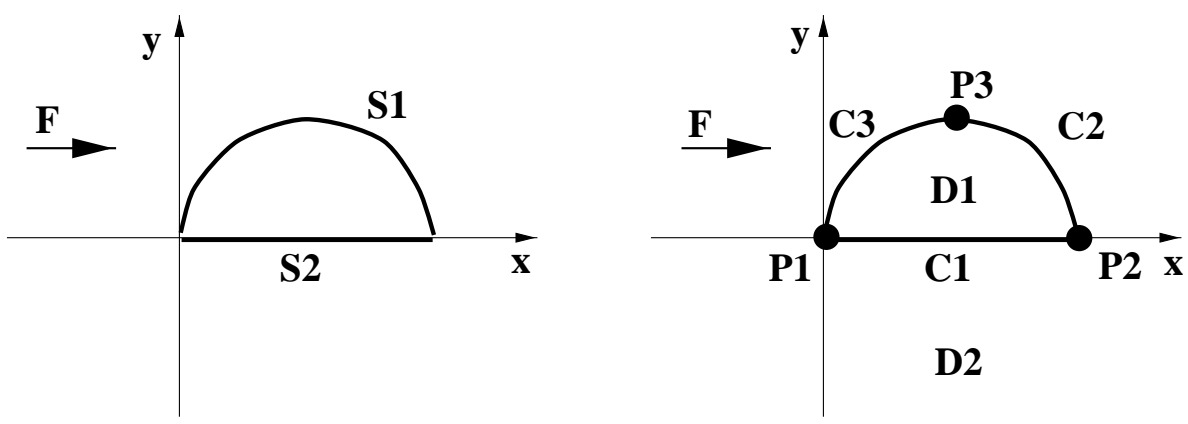

Fig. 2. Subanalytic stratification example

In view of the above properties we will restrict our study to hybrid systems for which the relevant sets are all relatively compact and subanalytic. 
Assumption 1 : For each discrete state $q$ the collection $\mathcal{A}_{q}$ consists of relatively compact subanalytic sets. In particular, we assume there exists a compact set $K$ such that if $A \in \mathcal{A}_{q}$ then $A \subseteq K$.

The partition $\mathcal{S}_{q}$ which serves as the initialization step of Algorithm 2 can now be assumed to be a subanalytic stratification compatible with $\mathcal{A}_{q}$ and the vector field $F(q, \cdot)$ (as given by Theorem 10).

The following proposition illustrates some of the good intersection properties that analytic curves have with subanalytic sets. The "finiteness" property indicated in the proposition makes it possible to define transitions between adjacent strata in a natural way.

Proposition 1. Let $I$ be an open interval, $M$ a real analytic manifold and $\gamma: I \rightarrow M$ a real analytic function. Let $\mathcal{S}$ be a $C^{\omega}$ stratification of $M$ by subanalytic sets If $[a, b] \subset I$ then there exists a finite partition $\left\{x_{1}, \ldots, x_{n}\right\}$ of $[a, b]$ with the property that for each $i=1, \ldots, n-1$ there exists a stratum $S_{i} \in \mathcal{S}$ such that $\gamma\left(\left(x_{i}, x_{i+1}\right)\right) \subseteq S_{i}$.

Proof. The family $\mathcal{I}=\left\{\gamma^{-1}(S) \cap[a, b]: S \in \mathcal{S}\right\}$ is a finite partition of $[a, b]$ by subanalytic sets. Each such set consists of a finite number of points and open intervals. Using all such points and the endpoints of such intervals gives the desired partition.

The following example shows the type of pathological situations that can be encountered if the assumption on subanalyticity is even slightly relaxed.

Example 4. Consider the stratification of $\mathbb{R}^{2}$ by the following five sets:

$$
\begin{aligned}
& S_{1}=\{(0,0)\} \\
& S_{2}=\left\{(x, y): x>0 \wedge y=x \sin \frac{1}{x}\right\} \\
& S_{3}=\left\{(x, y): x<0 \wedge y=x \sin \frac{1}{x}\right\} \\
& S_{4}=\left\{(x, y): x \neq 0 \wedge y>x \sin \frac{1}{x}\right\} \bigcup\{(0, y): y>0\} \\
& S_{5}=\left\{(x, y): x \neq 0 \wedge y<x \sin \frac{1}{x}\right\} \bigcup\{(0, y): y<0\}
\end{aligned}
$$

Notice that $S_{1}, S_{2}$ and $S_{3}$ form the graph of the function $f(x)=x \sin \frac{1}{x}$ $(f(0)=0)$, while $S_{4}$ and $S_{5}$ denote the region above and the below the graph, respectively. Each set is a $C^{\omega}$, embedded submanifold of $\mathbb{R}^{2}$ and they clearly satisfy the condition on the dimension of the strata in the closure of other strata. Finally, consider the constant vector field $F=\frac{\partial}{\partial x}$. Then the integral curve $\gamma$ of $F$ through $(0,0)$ is the $x$-axis (parameterized by $x$ itself). Therefore, the image by $\gamma$ of any interval containing 0 intersects both $S_{4}$ and $S_{5}$ an infinite number of times. This is reminiscent of the undesirable zeno property which allows an infinite number of switches in finite time. 


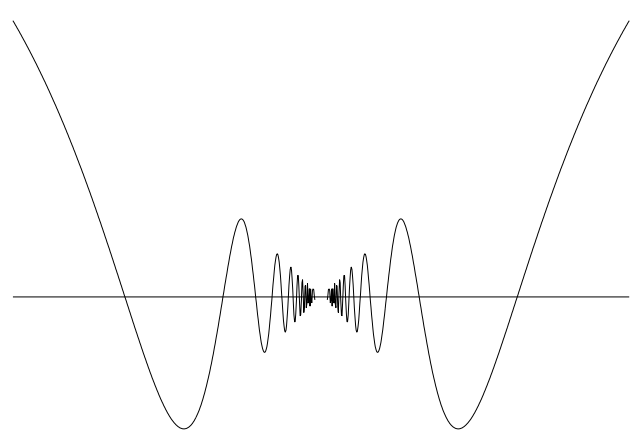

Fig. 3. Infinite crossings on a compact interval

Since the algorithm considers one discrete state at a time, we will simplify the notation by assuming that the discrete state $q$ is fixed and drop it as a subscript. In particular we will consider a vector field $F$ and a stratification $\mathcal{S}$ of $X_{C}$ by subanalytic sets as provided by Theorem 1 By $X_{C} / \sim$ we will mean the partition of $X_{C}$ induced by $\mathcal{S}$. We will denote by $\gamma_{x}$ the integral curve of $F$ which passes through $x$ at time 0 , i.e. with $\gamma_{x}(0)=x$.

We now proceed to formalize the notion of a discretization of the continuous transitions relative to a given partition $\mathcal{S}$. We do this mainly it simplifies the arguments in the proof of the main theorem (Theorem 2). In addition it supports the intuitive picture we have that a trajectory can be decomposed as a concatenation of pieces in each of the sets in $\mathcal{S}$.

Definition 5 (Transition relative to $\mathcal{S}$ : version 1). Given $x, y \in X_{C}$ we say $x \stackrel{\mathcal{S}}{\rightarrow} y$ iff there is $t>0$ such that $\gamma_{x}(t)=y$ and there exists $S \in \mathcal{S}$ such that $\gamma_{x}(s) \in S$ for $0<s<t$ and at least one of $x, y$ is in $S$.

To clarify this concept and to facilitate further discussions and proofs we introduce additional definitions.

Definition 6. Given two subsets $S_{1}, S_{2}$ of $X_{C}$, and a real analytic curve $\gamma$ : $I \rightarrow X_{C}$ where $I$ is an open interval, we say that $\gamma$ leaves $S_{1}$ through $S_{2}$ (or enters $S_{2}$ from $S_{1}$ ) if one of the following exiting conditions is satisfied:

E1 there exist $a, b \in I, a<b$, such that $\gamma(t) \in S_{1}$ for all $t \in(a, b)$ and $\gamma(b) \in S_{2}$ E2 there exist $a, b \in I, a<b$, such that $\gamma(a) \in S_{1}$ and $\gamma(t) \in S_{2}$ for all $t \in(a, b)$.

When $x \in S_{1}$ we say that $\gamma_{x}$ leaves $S_{1}$ trough $S_{2}$ if either $\boldsymbol{E} 1$ or $\boldsymbol{E} 2$ holds with $a=0$.

The following proposition is a simple application of Proposition 1 and shows that Definition [6] covers all possible "exiting" situations for strata of $\mathcal{S}$. 
Proposition 2. Let $S_{1} \in \mathcal{S}$ and $\gamma$ be as above. If there exists $t_{0}, t_{1} \in I$ such that $\gamma\left(t_{0}\right) \in S_{1}$ and $\gamma\left(t_{1}\right) \notin S_{1}$ then there exists a stratum $S_{2}\left(\neq S_{1}\right)$ such that either $\mathbf{E 1}$ or $\mathbf{E 2}$ holds.

It is clear from Definition [6] that in case $\mathbf{E} 1, S_{2} \cap \bar{S}_{1} \neq \emptyset$. By the properties of stratifications, we conclude $S_{2} \subset \bar{S}_{1}$ and $\operatorname{dim} S_{2}<\operatorname{dim} S_{1}$. Therefore, the flow exits the stratum $S_{1}$ though a stratum of lower dimension. Similarly in case E2, $S_{1} \subset \bar{S}_{2}$ and $\operatorname{dim} S_{1}<\operatorname{dim} S_{2}$ and the flow enters $S_{2}$ from a stratum of lower dimension. The following proposition further clarifies the possible exit situations.

Definition 7. We call a stratum $S \in \mathcal{S}$ tangential if the vector field $F$ is tangent to $S$ at every point of $S$. We call a stratum transversal otherwise.

Proposition 3. Let $S_{1}, S_{2}$ be strata in $\mathcal{S}$ and $\gamma$ an integral curve of $F$ which leaves $S_{1}$ through $S_{2}$. Then one (and only one) of the following holds:

1. condition $\mathbf{E} 1$ holds, $S_{1}$ is a tangential stratum and $S_{2}$ is a transversal stratum.

2. condition E2 holds, $S_{1}$ is a transversal stratum and $S_{2}$ is a tangential stratum.

We can now give the alternative definition of relative transitions.

Definition 8 (Transition relative to $\mathcal{S}$ : version 2). For each $x \in X_{C}$ let $S(x)$ denote the unique stratum in $\mathcal{S}$ which contains $x$. Given $x, y \in X_{C}$ we say $x \stackrel{\mathcal{S}}{\rightarrow} y$ iff $\gamma_{x}$ leaves $S(x)$ through $S(y)$.

It is clear from Proposition 1 that $x \stackrel{\tau}{\rightarrow} y$ iff there exist $x_{1}, \ldots, x_{n}$ such that $x \stackrel{\mathcal{S}}{\rightarrow} x_{1} \stackrel{\mathcal{S}}{\rightarrow} \ldots \stackrel{\mathcal{S}}{\rightarrow} x_{n} \stackrel{\mathcal{S}}{\rightarrow} y$. We will denote the Pre operator associated to $\stackrel{\mathcal{S}}{\rightarrow}$ by $\operatorname{Pre}_{\mathcal{S}}$. The above remark also implies that we can substitute $\operatorname{Pre}_{\mathcal{S}}$ for $\operatorname{Pre}_{\tau}$ in Algorithm 2 in the sense that if the algorithm terminates using Pre $_{\mathcal{S}}$ then it also terminates when using $\operatorname{Pre}_{\tau}$.

As the stratification Theorem 1 shows, issues of transversality of trajectories can be analyzed within the context of subanalytic sets and analytic vector fields. However, the study of continuous transitions requires that we investigate the global behavior of trajectories. In general, trajectories of analytic vector field$\mathrm{s}$ (and much less their full flows) are not subanalytic. Identifying vector fields whose flows belong to a suitable class is the main obstacle in the study of bisimulations of hybrid systems. Recent developments in logic model theory provide some answers as well as suggest the proper context in which to carry on further studies.

\section{Model Theory}

Model theory studies structures through properties of their definable sets (see [14]23] for general background). The basic structures of interest for this 
paper are that of the real numbers as a complete ordered field, symbolized by $(\mathbb{R},+,-, \times,<, 0,1)$, and its extensions. Every such structure $L$ has an associated language $\mathcal{L}$ of formulas. The (first order) formulas over $\mathcal{L}$ are the wellformed logical expressions obtained by using logical connectives, quantifiers $\exists$ $\forall$, real numbers as constants, the operations of additions and multiplication, and the relations $<$ and $=$ (quantification is allowed over variables). All formulas will be interpreted over the real numbers. A definable set in the language $\mathcal{L}$ (or of the structure $L$ ) is a subset of $\mathbb{R}^{n}$ (for some $n$ ) of the form $\left\{\left(a_{1}, \ldots, a_{n}\right) \in \mathbb{R}^{n}: \Phi\left(a_{1}, \ldots, a_{n}\right)\right\}$, where $\Phi\left(x_{1}, \ldots, x_{n}\right)$ is a formula in $\mathcal{L}$ and $x_{1}, \ldots, x_{n}$ are free (i.e. not quantified) variables in $\Phi$. A function $f$ is definable if its graph is a definable set.

While many of the concepts here apply to more general structures, in all that follows we consider only structures over the real numbers.

Definition 9. The theory of $\mathcal{L}$ is o-minimal ("order minimal") if every definable subset of $\mathbb{R}$ is a finite union of points and intervals (possibly unbounded).

Tarski [22] was interested in the extension of the theory of the real numbers by the exponential function, $(\mathbb{R},+,-, \times,<, 0,1$, exp) (i.e., there is an additional symbol in the language for the exponential function). We denote this structure by $\mathbb{R}_{\text {exp }}$. While such theory does not admit elimination of quantifiers, it was shown in 25] that such theory is model complete, which in turns implies that it is o-minimal. Another important extension is obtained as follows. Assume $f$ is a real-analytic function in a neighborhood of the cube $[-1,1]^{n} \subset \mathbb{R}^{n}$. Let $\hat{f}: \mathbb{R}^{n} \rightarrow \mathbb{R}$ be the function defined by

$$
\hat{f}(x)= \begin{cases}f(x) & \text { if } x \in[-1,1]^{n} \\ 0 & \text { otherwise }\end{cases}
$$

We call such functions restricted analytic functions. The structure $\mathbb{R}_{\text {exp,an }}=$ $(\mathbb{R},+,-, \times,<, 0,1, \exp ,\{\hat{f}\})$ is then an extension of $\mathbb{R}_{\exp }$ where there is a symbol for each restricted analytic function. One reason this structure is relevant for this paper is that all relatively compact subanalytic sets are definable in $\mathbb{R}_{\text {exp,an }}$. Moreover, if $F$ is a linear vector field in $\mathbb{R}^{n}$ with real eigenvalues, then the

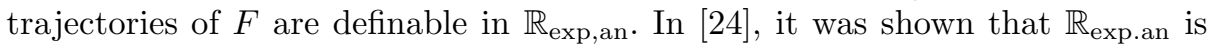
also o-minimal. Finally, there are a few consequences of o-minimality that are crucial for our results. We list them below under one proposition. The proofs are contained in the various references mentioned above.

Proposition 4. Assume $L$ is an o-minimal structure. Then

1. Any definable set has a finite number of connected components, each of which is a definable set.

2. If $A$ is definable, then so is its (topological) closure. Moreover, $\operatorname{dim} \operatorname{Fr}(A)<$ $\operatorname{dim} A$, where $\operatorname{Fr}(A)=\bar{A} \backslash A$ is the frontier of $A$ and the dimension of a set $B \subset \mathbb{R}^{n}$ is the maximum integer $d$ for which there is an embedded $C^{1}$ manifold of $\mathbb{R}^{n}$ contained in $B$. 
3. Given definable sets $A_{1}, \ldots, A_{k}$ in $\mathbb{R}^{n}$ (and for any integer $p$ ), there is a finite $C^{p}$ stratification of $\mathbb{R}^{n}$ compatible with $\left\{A_{1}, \ldots, A_{k}\right\}$. In fact, for the structure $\mathbb{R}_{\text {exp,an }}$ the strata are definable (real) analytic manifolds.

We are now ready to apply these results to prove that Algorithm 2 terminates for certain classes of planar systems.

\section{$6 \quad$ Finiteness Results}

In this section we use the model theoretic tools of Section 5 in order to obtain classes of systems for which the Bisimulation Algorithm of Section 3 terminates.

Recall that given the family of sets $\mathcal{A}$ as in Assumption 1, and the vector field $F$ we first obtain a stratification $\mathcal{S}$ compatible with $\mathcal{A}$ as given by Theorem 1 . We will also assume that $\mathcal{S}$ is compatible with a compact subanalytic set $K$ which contains all sets in $\mathcal{A}$. We define $\mathcal{S}_{K}=\{S \in \mathcal{S}: S \cap K \neq \emptyset\}$ (which is therefore finite).

Theorem 2. Let $X_{C}=\mathbb{R}^{2}, F$ be the linear vector field $A x$ and assume that the eigenvalues of $A$ are real. Then the bisimulation algorithm for hybrid systems (Algorithm 2), initialized with $\mathcal{S}_{K}$, terminates.

Proof. We will consider the case when the origin is the only equilibrium of $F$. (The other cases require minor modifications.) We assume without loss of generality that $\{(0,0)\} \in \mathcal{S}_{\mathcal{K}}$.

As indicated in Section 3 it suffices to study only the evolution of the continuous variables and use $\operatorname{Pre}_{\mathcal{S}}$ in Algorithm 2. To simplify notation we will simply refer to it as Pre. In order to show that the bisimulation algorithm terminates we will construct a finite refinement of $\mathcal{S}_{K}$ which is "invariant" under the Pre operation and which is a refinement of $X_{C} / \sim$ at each step.

For each stratum $S \in \mathcal{S}_{K}$ with $(0,0) \in \bar{S}$ we consider the set

$$
S_{\infty}=\left\{x \in S: \forall t \geq 0 \quad \gamma_{x}(t) \in S\right\}
$$

As mentioned earlier, since the eigenvalues of $A$ are real, the flow of $F$, $\Phi(x, t)=\gamma_{x}(t)=e^{t A} x$ is definable in $\mathbb{R}_{\text {exp, an }}$ (the entries in $e^{t A}$ involve polynomials and real exponential functions). Therefore, the set $S_{\infty}$ is definable. For each stratum $T$ of dimension one with $T \subset \bar{S}, T \neq S$, we consider the set

$$
T_{*}=\left\{x \in T: \gamma_{x} \text { leaves } T \text { through } S_{\infty}\right\}
$$

The set $T_{*}$ is also definable in $\mathbb{R}_{\text {exp,an }}$ and therefore can be written as a finite, disjoint union of definable sets each of which is either a point or homeomorphic to an open interval. We may assume, by refining the original $\mathcal{S}_{K}$ if necessary that the finitely many points in the decomposition of $T_{*}$ are already strata of $\mathcal{S}_{K}$.

For each $x \in \mathbb{R}^{2}$ let $\Gamma_{x}$ denote the trajectory of $F$ passing through $x$, that is

$$
\Gamma_{x}=\left\{\gamma_{x}(t): t \in \mathbb{R}\right\} .
$$


For each stratum $S \in \mathcal{S}$ and $x \in S$, let $\Gamma_{x}(S)$ denote the connected component of $\Gamma_{x} \cap S$ which contains $x$. It is clear, from the definition of $S_{\infty}$, that if $x \in S_{\infty}$ then $\Gamma_{x}(S) \subset S_{\infty}$. From this it follows that if $x \in T$ and $\gamma_{x}$ leaves $T$ through $S$ then $\gamma_{x}$ either leaves $T$ though $S_{\infty}$ or leaves $T$ through $S \backslash S_{\infty}$.

Let $\left\{p_{1}\right\}, \ldots,\left\{p_{l}\right\}$ be all the 0 -dimensional strata of $\mathcal{S}_{K}$. Notice that for each $i, j$, if $\Gamma_{p_{i}} \cap \Gamma_{p_{j}} \neq \emptyset$, then $\Gamma_{p_{i}}=\Gamma_{p_{j}}$. We will eliminate redundancies and assume that the $\Gamma_{p_{i}}$ are pairwise disjoint. For each set $S \in \mathcal{S}_{K}$ and each $\Gamma_{p_{i}}$, the sets $S \cap \Gamma_{p_{i}}$ and $S \backslash \cup_{i} \Gamma_{p_{i}}$ are definable in $\mathbb{R}_{\text {exp,an }}$ (Intuitively, these sets are partitions of $S$ "in the direction of the flow of $F$ "). By o-minimality, we get that each such set has a finite number of connected components. Let $\mathcal{B}$ denote the (finite) collection of all such connected components. The collection $\mathcal{B}$ is then a partition of $K$ compatible with $\mathcal{S}$ (every set in $\mathcal{S}$ is a union of sets in $\mathcal{B}$ ).

Claim: At each step of the bisimulation algorithm, $\mathcal{B}$ is compatible with $M / \sim$.

The claim shows that $\mathcal{B}$ is finer than all partitions obtained at each step. Since $\mathcal{B}$ is finite, this proves that the algorithm terminates.

To prove the claim we first show that if $B_{i} \in \mathcal{B}$ for $i=1, \ldots, n$ then

$$
\operatorname{Pre}\left(\cup B_{i}\right)=\cup \operatorname{Pre}\left(B_{i}\right)
$$

We will call a set $B \in \mathcal{B}$ tangential if $B$ is contained in a tangential stratum of $\mathcal{S}$ (i.e. $B$ is a connected component of either $S \cap \Gamma_{q}$ or $S \backslash \cup \Gamma_{p_{i}}$ with $S$ tangential). The set $B$ will be called transversal otherwise. Notice that if $B$ is tangential and $x \in B$ then $\Gamma_{x}(S(x)) \subset B$.

Let $x \in \operatorname{Pre}\left(B_{i}\right)$ for some $i=1, \ldots, n$ and $x \notin B_{i}$. Suppose $\gamma_{x}(t) \in S(x)$ for $0 \leq t<\delta$ and $\gamma_{x}(\delta) \in B_{i}$ (i.e. exit condition E1). In particular, $S(x)$ is a tangential stratum. If $\gamma_{x}(t) \notin \cup B_{i}$ for $t<\delta$, then $x \in \operatorname{Pre}\left(\cup B_{i}\right)$. If $\gamma_{x}(t) \in \cup B_{i}$ for some $t<\delta$, then for some $j, B_{j}$ is tangential, so $\Gamma_{x}(S(x)) \subset B_{j}$ and $x \in$ $\operatorname{Pre}\left(\cup B_{i}\right)$. If, instead, $\gamma_{x}(t) \in B_{i}$ for $0<t<\delta$ (exit condition E2), then clearly $x \in \operatorname{Pre}\left(\cup B_{i}\right)$.

Conversely, let $x \in \operatorname{Pre}\left(\cup B_{i}\right)$. If $\gamma_{x}(t) \in S(x)$ for $0 \leq t<\delta, \gamma_{x}(\delta) \in \cup B_{i}$, let $i_{0}$ be such that $\gamma_{x}(\delta) \in B_{i_{0}}$. Then $x \in \operatorname{Pre}\left(B_{i_{0}}\right) \subset \cup \operatorname{Pre}\left(B_{i}\right)$. If, instead, $\gamma_{x}(t) \in \cup B_{i}$ for $0<t<\delta$, then there is a $\delta_{0}>0$ and a $B_{i_{0}}$ which contains $\gamma_{x}(t)$ for $0<t<\delta_{0}$ (here we used o-minimality again to conclude that $\Gamma_{x}$ intersects each $B_{i}$ in a finite disjoint union of points and arcs). Therefore, $x \in \operatorname{Pre}\left(B_{i_{0}}\right)$. This conclude the proof of (4).

By construction, $\mathcal{B}$ is compatible with $\mathcal{S}_{K}$. At each step of the bisimulation algorithm we need to show that if $B=\cup_{i=1}^{n} B_{i}$ and $B^{\prime}=\cup_{j=1}^{m} B_{i}^{\prime}$ with $B_{i}, B_{i}^{\prime} \in \mathcal{B}$ then $B \cap \operatorname{Pre}\left(B^{\prime}\right)$ is again a finite union of sets in $\mathcal{B}$. Based on (4) it will suffice to show that for $B, B^{\prime} \in \mathcal{B}$, either $B \cap \operatorname{Pre}\left(B^{\prime}\right)=\emptyset$ or $B \cap \operatorname{Pre}\left(B^{\prime}\right)=B$.

We consider several cases. The set $B$ is of one of the two forms: (a) a connected component of $S \cap \Gamma_{p_{i}}$, or (b) a connected component of $S \backslash \cup \Gamma_{p_{i}}$.

If $S$ is 0 -dimensional there is nothing to show because $B$ contains a single point.

If $S$ is 1-dimensional and $B$ is of type (a), then either $S$ is transversal and $B$ consists of a single point or $S$ is tangential and so $B=\Gamma_{x}(S)$ for any $x \in B$. 
The first case is again clear. In the second case, if there is $x \in B \cap \operatorname{Pre}\left(B^{\prime}\right)$ then there exists $\delta>0$ such that $\gamma_{x}(t) \in S$ for $0 \leq t<\delta$ and $\gamma_{x}(\delta) \in B^{\prime}$. But then for all $y \in \Gamma_{x}(S), \gamma_{y}$ leaves $S$ through $B^{\prime}$. So $B=\Gamma_{x}(S) \subset \operatorname{Pre}\left(B^{\prime}\right)$.

If $S$ is 1-dimensional and $B$ is of type (b) then we again consider separately the cases when $S$ is tangential and when $S$ is transversal. In the first case we proceed as before. Assume now, that $S$ is transversal. Notice that if $x \in B \cap$ $\operatorname{Pre}\left(B^{\prime}\right)$ then $\Gamma_{x}$ intersects both $B$ and $B^{\prime}$. Therefore $B^{\prime}$ is also a connected component of $S^{\prime} \backslash \cup \Gamma_{p_{i}}$ (for some $S^{\prime}$ ). By transversality, $\gamma_{x}$ leaves $S$ intro $S^{\prime}$ under exit condition E2 and so $S \subset \operatorname{Fron}\left(S^{\prime}\right)\left(=\overline{S^{\prime}} \backslash S^{\prime}\right)$ and $S^{\prime}$ is 2-dimensional. By continuity of the flow of $F$, there is an open neighborhood $N$ of $x$ such that for $y \in N \cap B, \gamma_{y}$ leaves $S$ through $S^{\prime}$. Moreover, since there are finitely many $\Gamma_{p_{i}}$ we may assume (by taking $N$ smaller) that $\gamma_{y}$ leaves $S$ through $B^{\prime}$. We have then showed that the set $E=\left\{x \in B: \gamma_{x}\right.$ leaves $\mathrm{S}$ through $\left.B^{\prime}\right\}$ is open in $B$. Suppose $E \neq B$. Then there is $y \in B$ in the frontier of $E$. We can find a neighborhood $W$ of $y$ such that $W \cap \Gamma_{p_{i}}=\emptyset$ for all $i$. Since $S^{\prime}$ is open in $\mathbb{R}^{2}$, and $S$ is transversal, we can find a neighborhood $W_{0} \subset W$ of $y$ and $\varepsilon>0$ such that for $z \in W_{0} \cap S$ and $0<t<\varepsilon$ we have $\gamma_{x}(t) \in W \cap S^{\prime}$. But then every such $z$ belongs to $E$. This contradicts the fact that $y$ is a frontier point. Therefore, $E$ is also closed in $B$ and so it must equal $B$ (since $B$ is connected). We conclude in this case that $B=B \cap \operatorname{Pre}\left(B^{\prime}\right)$.

There is only one case remaining: $S$ of dimension 2 (and hence tangential). If $B$ is of type (a) then $\Gamma_{x}(S)=B$ and we are done as before.

Assume then that $B$ is a connected component of $S \backslash \cup \Gamma_{p_{i}}, B^{\prime}$ a connected component of $S^{\prime} \backslash \cup \Gamma_{p_{i}}, S^{\prime}$ is transversal, and $\operatorname{dim} S^{\prime}=1$. (The case with $S^{\prime}$ 0 -dimensional is excluded since in that case $S^{\prime} \cap \Gamma_{p_{i}} \neq \emptyset$ for some $i$.)

Let $x \in B \cap \operatorname{Pre}\left(B^{\prime}\right)$ and assume there is $y \in B \backslash \operatorname{Pre}\left(B^{\prime}\right)$. We want to show that this leads to a contradiction. Let $\alpha:[0,1] \rightarrow B$ be a curve connecting $x$ to $y$. Let $t_{0}$ be the smallest $t \in[0,1]$ such that $\gamma_{\alpha(t)}(s) \notin B^{\prime}$ for some $s>0$. If $\gamma_{\alpha\left(t_{0}\right)}(s) \in S$ for all $s>0$ then $\alpha\left(t_{0}\right) \in S_{\infty}$. By the choice of $t_{0}$ we in fact have $\alpha\left(t_{0}\right) \in \Gamma_{p_{0}}$ for some $p_{0}$ (see the initial subdivision caused by $S_{\infty}$ ). But this contradicts the fact that $B$ is of type (b). Assume then that $\gamma_{\alpha\left(t_{0}\right)}(s) \notin S$ for some $s>0$. For each $t \in\left[0, t_{0}\right]$ let $s(t)$ be the smallest $s$ such that $\gamma_{\alpha(t)}(s) \notin S$. For each $t \in\left[0, t_{0}\right]$ set $p(t)=\gamma_{\alpha(t)}(s(t))$. There are two possibilities: either $p\left(t_{0}\right) \in S^{\prime}$ or $p\left(t_{0}\right) \in \overline{S^{\prime}} \backslash S$.

In the first case choose a local chart $(N, \varphi)$ centered at $p\left(t_{0}\right)$ so that in $\varphi$ coordinates we have $N \cap S^{\prime}=N \cap B^{\prime}=\{(x, 0)\}$ and $N \cap S=\{(x, y): y>$ $0\}$ (therefore $F$ points into the lower half plane at every point of $N \cap B^{\prime}$. By continuity of the flow and transversality, we still have that $\gamma_{\alpha(t)}$ crosses $N \cap B^{\prime}$ from the upper to the lower half plane for $t_{0}<t<t_{0}+\epsilon$. But this contradicts the choice of $t_{0}$.

In the second case, we have $p\left(t_{0}\right) \in \Gamma_{q_{0}}$ for some $q_{0}$. But this contradicts the fact that $B$ is of type (b).

All this implies that every $y$ in $B$ must also be in $\operatorname{Pre}\left(B^{\prime}\right)$. That is, $B=$ $B \cap \operatorname{Pre}\left(B^{\prime}\right)$. This concludes the proofs of the claim and the theorem. 
As the proof above suggests the termination of the algorithm depends on the fact that the integral curves of the vector field intersects relatively compact subanalytic sets in at most finitely many points. This allows us to get the following generalization.

Theorem 3. If $F$ is an analytic vector field in $\mathbb{R}^{2}$ which admits an analytic family of first integrals, then the bisimulation algorithm terminates. (Here, by an analytic family of first integrals we mean a non-constant (real) analytic function $f: \mathbb{R}^{2} \rightarrow \mathbb{R}$ such that for each trajectory $\gamma$ of $F$ the function $f(\gamma(t))$ is constant.)

Proof. Notice that each level curve of $f$ is an analytic set and therefore its intersection with any relatively compact definable set (in $\mathbb{R}_{\text {exp,an }}$ ) is definable in $\mathbb{R}_{\text {exp,an }}$. The proof then follows the lines of the previous one but replacing the sets $\Gamma_{p_{i}}$, with the corresponding level set of $f$ (level sets of $f$ are at most 1-dimensional since $f$ is not constant on any open set).

Corollary 1. If $F$ is a linear vector field in $\mathbb{R}^{2}$ with purely imaginary eigenvalues and $\mathcal{S}_{K}$ is as in the theorem, then the bisimulation algorithm terminates.

Proof. Unless $A=0$, in which case the result is trivial, there exists an (invertible) matrix $P$ such that $\|P x\|^{2}$ is constant along trajectories of $F$.

Corollary 2. If $F$ is an analytic Hamiltonian vector field in $\mathbb{R}^{2}$ and $\mathcal{S}_{K}$ is as above, then the bisimulation algorithm terminates.

Proof. The Hamiltonian is constant along the trajectories.

Remark 1. As is clear from the proofs above, the key is that all the objects involved (the vector field $F$, the initial family of sets, the flow of $F$ ) be definable in some o-minimal extension of the field of real numbers. We presented above just two specific instances of such a situation which can be easily characterized. A more recent o-minimal extension of the reals, by so called Pfaffian functions, was found in [26].

\section{Conclusions}

In this paper, we presented new classes of planar hybrid systems with finite bisimulations. This was achieved by combining the geometric framework of subanalytic sets with model theoretic concepts from mathematical logic. The mathematical tools used in this paper provide the natural platform for studying decidability of computational algorithms for hybrid systems.

Issues for future research, include the extention of these results to $\mathbb{R}^{n}$ as well as to hybrid systems whose relevant sets and flows are definable in o-minimal structures. In addition, the issue of decidability requires not only termination of the bisimulation algorithm but also constructive decision methods for each step. 
Even though decision methods exist for $(\mathbb{R},+,-, \times,<, 0,1)[22$, it is not known if the theory of $\mathbb{R}_{\exp }$ is decidable, although in [16] it was shown that it would be a consequence of Schanuel's conjecture in number theory. The results we obtained in this paper suggest how to find some restricted classes of vector fields for which the algorithm is constructive. Indeed, if all the relevant sets are semialgebraic (for example if $F$ is a Hamiltonian vector field on the plane with a polynomial Hamiltonian and the initial conditions, guards, etc., are semialgebraic), then they are definable in $(\mathbb{R},+,-, \times,<, 0,1)$. Such a decidability result is obtained in 9 .

\section{References}

1. R. Alur, C. Coucoubetis, N. Halbwachs, T.A. Henzinger, P.H. Ho, X. Nicolin, A. Olivero, J. Sifakis, and S. Yovine, The algorithmic analysis of hybrid systems, Theoretical Computer Science 138 (1995), 3-34.

2. R. Alur and D.L. Dill, A theory of timed automata, Theoretical Computer Science 126 (1994), 183-235.

3. R. Alur, T.A. Henzinger, and E.D. Sontag (eds.), Hybrid systems III, Lecture Notes in Computer Science, vol. 1066, Springer-Verlag, 1996.

4. P. Antsaklis, W. Kohn, A. Nerode, and S. Sastry (eds.), Hybrid systems II, Lecture Notes in Computer Science, vol. 999, Springer-Verlag, 1995.

5. P. Antsaklis, W. Kohn, A. Nerode, and S. Sastry (eds.), Hybrid systems IV, Lecture Notes in Computer Science, vol. 1273, Springer-Verlag, 1997.

6. E. Bierstone and P.D. Milman, Semianalytic and subanalytic sets, Inst. Hautes Études Sci. Publ. Math. 67 (1988), 5-42.

7. P.E. Caines and Y.J. Wei, The hierarchical lattices of a finite state machine, Systems and Control Letters 25 (1995), 257-263.

8. _ Hierarchical hybrid control systems: A lattice theoretic formulation, IEEE Transactions on Automatic Control : Special Issue on Hybrid Systems 43 (1998), no. 4, 501-508.

9. K. Cerans and J. Viksna, Deciding reachability for planar multi-polynomial systems, Hybrid Systems III (R. Alur, T. Henzinger, and E.D. Sontag, eds.), Lecture Notes in Computer Science, vol. 1066, Springer Verlag, Berlin, Germany, 1996, pp. 389400.

10. R. L. Grossman, A. Nerode, A. P. Ravn, and H. Rischel (eds.), Hybrid systems, Lecture Notes in Computer Science, vol. 736, Springer-Verlag, 1993.

11. T.A. Henzinger, Hybrid automata with finite bisimulations, ICALP 95: Automata, Languages, and Programming (Z. Fülöp and F. Gécseg, eds.), Springer-Verlag, 1995, pp. 324-335.

12. T.A. Henzinger, P.W. Kopke, A. Puri, and P. Varaiya, What's decidable about hybrid automata?, Proceedings of the 27th Annual Symposium on Theory of Computing, ACM Press, 1995, pp. 373-382.

13. H. Hironaka, Subanalytic sets, In Number Theory, Algebraic Geometry, and Commutative Algebra, in honor of Y. Akizuki, Kinokuniya Publications, 1973, pp. 453493.

14. W. Hodges, A shorter model theory, Cambridge University Press, 1997. 
15. G. Lafferriere, G.J. Pappas, and S. Sastry, Subanalytic stratifications and bisimulations, Hybrid Systems : Computation and Control (T. Henzinger and S. Sastry, eds.), Lecture Notes in Computer Science, vol. 1386, Springer Verlag, Berlin, 1998, pp. 205-220.

16. A. Macintyre and A.J. Wilkie, On the decidability of the real exponential field, Kreiseliana: About and around Georg Kreisel, A.K. Peters, 1996, pp. 441-467.

17. O. Maler (ed.), Hybrid and real-time systems, Lecture Notes in Computer Science, vol. 1201, Springer-Verlag, 1997.

18. G.J. Pappas, G. Lafferriere, and S. Sastry, Hierarchically consistent control systems, Proceedings of the 37th IEEE Conference in Decision and Control, Tampa, FL, December 1998, Submitted.

19. A. Puri and P. Varaiya, Decidability of hybrid systems with rectangular differential inclusions, Computer Aided Verification, 1994, pp. 95-104.

20. Héctor J. Sussmann, Subanalytic sets and feedback control, Journal of Differential Equations 31 (1979), no. 1, 31-52.

21. _ Real-analytic desingularization and subanalytic sets: An elementary approach, Transactions of the American Mathematical Society 317 (1990), no. 2, 417-461.

22. A. Tarski, A decision method for elementary algebra and geometry, second ed., University of California Press, 1951.

23. D. van Dalen, Logic and structure, third ed., Springer-Verlag, 1994.

24. L. van den Dries and C. Miller, On the real exponential field with restricted analytic functions, Israel Journal of Mathematics 85 (1994), 19-56.

25. A. J. Wilkie, Model completeness results for expansions of the ordered field of real numbers by restricted pfaffian functions and the exponential function, Journal of the American Mathematical Society 9 (1996), no. 4, 1051-1094.

26. A.J. Wilkie, A general theorem of the complement and some new o-minimal structures, Preprint, 1997. 